\title{
Disagreement Constrained Action Selection in Participatory Portfolio Decision Analysis
}

\author{
Tobias Fasth, Aron Larsson, and Maria Kalinina
}

\begin{abstract}
In some portfolio decision problems it is not possible or interesting to constrain portfolios with a monetary budget. Instead it might be of interest to investigate how disagreement among a group of decision makers or stakeholders can be used as a constraint, and how this affects the portfolio composition. In this paper we present complementary decision evaluation methods for group portfolio decision analysis in situations where the stakeholders have conflicting preferences. The approach supports the analysis of a portfolio of planned actions in urban planning when a large group of stakeholders have inconsistent opinions with respect to the performance of each action. The group of stakeholders is, for each criterion, partitioned into two disagreeing groups based upon their views on the actions' performance. The distance between these two groups is then measured. An action's aggregated disagreement taking into account all criteria is then used as the action's associated resource constraint, and portfolios can be generated by solving a sequence of Knapsack problems. The robustness of the portfolios can be further evaluated with an a priori sensitivity analysis. The suggested approach supports decision makers by elucidating how the portfolio composition changes when the actions' aggregated disagreement increases.
\end{abstract}

Index Terms-E-Participation, e-democracy, urban planning, portfolio decision analysis, multi-criteria decision analysis, disagreement, consensus.

\section{INTRODUCTION}

This paper presents complementary decision evaluation concepts for group portfolio decision analysis. The rationale behind the concepts put forward is based on the existence of multi-criteria decision problems where the available actions are not mutually exclusive, and where there is a strong need for modeling and analysis of several stakeholder groups with differing preferences, cf. [1], [2]. Decision makers and/or stakeholders with differing preferences may lead to situations involving conflicts, which in turn may cause severe delays in the decision-making and planning process. Two recent examples of this within the context of urban planning are the development plans of Husby, a suburb of Stockholm, where the plans were delayed for several years [3], and the Swedish municipality Nacka where three infrastructure decision problems were delayed for several years [1], [2].

Manuscript received October 13, 2015; revised December 20, 2015.

Tobias Fasth and Aron Larsson are with the Department of Computer and Systems Sciences, Stockholm University, Kista, Sweden (e-mail: \{fasth, aron\}@dsv.su.se).

Maria Kalinina is with the Department of Information and Communications Systems, Mid Sweden University, Sundsvall, Sweden (email:mariak@dsv.su.se).
One way of approaching these multi-stakeholder problems with multiple non-mutually exclusive actions is to treat them as Portfolio Decision Analysis (PDA) problems. A portfolio typically consists of a set of planning actions, where each action has a value and an associated resource claim, such as a cost. The portfolio is then constrained by an overall cost budget, and possibly with additional constraints as well. This means that not all actions can be included in the portfolio, see e.g. [4]-[9].

However, in some situations it is not feasible to assign a cost to each action. Besides lack of cost information, it may sometimes not be ideal to use cost budget as the constraining resource. For instance, in previous research [10] we have noticed situations where the decision makers want to identify a subset of all tentative actions. These tentative actions are not necessarily described in detail, and properties such as timeframes or costs are absent. The interests here is rather to avoid time-consuming conflicts by,

1) investigate the stakeholders' disagreement with regard to each action, and

2) investigate how portfolios can be generated that elucidate how conflicting preferences affect the portfolio composition

To facilitate the decision process in such situation, each action should be associated with an indicator of its level of controversy. This "disagreement measure" should quantify the stakeholders' disagreement on that action. This information enables the selection of a portfolio of actions where the levels of total disagreement are not too high.

By using PDA techniques on group decision-making problems in the analysis of several combinations of several projects instead of analyzing them individually, increase the chances for compromises [11]. Different PDA techniques have been suggested, e.g., the Robust Portfolio Modeling (RPM) [4], [5] method which solves a multi-objective zeroone linear programming problem in the generation of portfolios. The method handles incomplete project scores with regard to each criterion, project costs and criterion weights. In [12] a group PDA method, which supports the development of shared action agendas of topics are developed. The topic, in their context, is a tentative proposal as opposed to an alternative in classical decision analysis. The method handles incomplete criterion weights and topic values for each criterion. Another method is PDELTA [8], [9], which is a PDA extension of the DELTA method, see e.g., [13], [14]. PDELTA handles incomplete project utilities for each criterion, project costs and criterion weights, and generates portfolios by solving a Knapsack problem. Moreover, the PROBE method [7], handles imprecise action values for each criterion, action costs and criterion weights. 
PROBE solves a Knapsack problem in the generation of portfolios.

Although previous PDA methods have constrained portfolios with resources such as a budget and other constraints e.g., [4], [5], [7]-[9], [12], to the best of our knowledge, no studies have used stakeholder disagreement as a constraint. We suggest a method for portfolio decision analysis, to be used in situations with multiple stakeholders, multiple actions, and when it is important to elucidate how disagreement between stakeholders affects the portfolio composition.

With respect to group decisions, Ref. [15] discussed multiple criteria approaches to group decision and negotiation. The goal of group decision and negotiation is to provide suitable procedures for decision-making, and to study the many issues, which may occur. A group decision problem is associated with several interested parties where the goal is to make a responsible choice, as opposed to negotiation, where the concerned parties may not be able to make final choice.

Further, [15] described, that the use of multi-criteria decision analysis (MCDA) techniques in group decision and negotiation presupposes that the sets of alternatives and criteria are given, and preference elicitation with succeeding aggregation of preferences can be performed by some welldeveloped MCDA technique. Direct application of MCDA techniques may not be possible due to the absence of unified collective preference. They recommended that criteria and individuals should be considered as separate entities to avoid well-known voting paradoxes. Group decision support methods, according to their opinion, can be divided into two categories. The first category is based on procedures, and the second is based on optimization and aggregation. With respect to the first category, it is the interaction between the decision maker and the group that enables the generation of new ideas and minimizes disagreement between groups. By contrast, the second category does not require any interaction between a decision maker and members of groups. It rather implies the design and implementation of an optimization model, where various aggregation approaches can be based on e.g., fuzzy logic, interval relations and probability, and the Dempster-Shafer theory.

Ref. [16] described how a multi-phase approach for flat sharing and how it was applied in a case study. The purpose of this multi-criteria approach is to help partners in location problems. The approach involves the following five steps; i) to use brainstorming while structuring the problem, ii) an individual ranking, iii) a grouping of partners by a hierarchical clustering, iv) a ranking of each group and v) negotiation. In the first phase, brainstorming can be adopted in order to generate a list of alternatives. In the second phase, the PROMETHEE method was used to individually rank the alternatives. They mention that their multi-criteria method does not require a normalization of scores, which in turn can lead to different ranking. However, this method requires a definition of a preference function by a decision maker. In the third phase, the partners are divided into smaller classes according to similarity of preferences. Both hierarchical and the partitional clustering algorithms can be used in the grouping phase. In the fourth phase, a group ranking of each group is obtained by using PROMETHEE II where each decision maker was given the same weight. Finally, in the fifth phase, negotiation, e.g. a Geographical Information Systems may aid the process.

Ref. [12] suggested a method for portfolio decision analysis with the aim of guiding the development of agendas in presence of incomplete group preferences. They built a portfolio model for the development of shared action agendas. In their study, the process of developing agendas is supported by MCDA techniques, which allow taking into consideration preferences of group members. The multicriteria framework is based on the additive multi-attribute value function. Incomplete information about criterion weights, scores, and group weights are modeled by set inclusion. The set inclusion implies the set feasible scores, the set of criterion weights and the set of group weights. The information about scores is expressed by interval statements and preference statements which are converted into sets of linear constraints on weight. Further, they suggested the use of four performance measures in order to compare nondominated portfolios in the view of acceptability of topics to various group members. These performance measures are minimum value, maximum regret value, acceptability index, and weak acceptability index. Computation of the nondominated portfolios set requires solving a multi-objective zero-one linear programming problem, where the objectives are the number of extreme points of auxiliary variables' set. The suggested framework is an iterative and interactive process allowing implementing the group members' preferences. Moreover, the framework is able to demonstrate the influence of preferences on the decision recommendation. The interactive process starts with determination and evaluation of action topics by stakeholders through interactive workshops or using a webplatform. The negotiation phase of the interactive process is based on an analysis of individual and group non-dominated portfolios, a creation of the set of recommended portfolios and subsequently discarding some portfolios from this set. Non-dominated portfolios with a high weak acceptability index are possible candidates since these portfolios are in core or borderline for many group members. Authors conclude that developed methods for agenda building processes are transparent at the level of individual group members as well as at the level of the whole group. Additionally, the overall accessibility of portfolios can be examined from various points of view through portfoliolevel performance measures.

\section{A METHOD FOR PARTICIPATORY PDA}

\section{A. Eliciting Stakeholder Preferences}

In our approach, the stakeholders' preferences are elicited by using a web-based questionnaire. We assume that; i) all stakeholders are equally important, ii) the stakeholders' preferences are independently stated, iii) the stakeholders' preferences are not influenced by other stakeholders, iv) that the questionnaire is self-explanatory. The questionnaire consists of a set of focus areas, each consisting of a set of actions. A focus area is an area of improvement, and can be considered as one criterion. The actions under a focus area are the suggested actions that may improve it. For instance, 
in the area "Development", possible actions are, "Build apartments in the city center", and "Build apartments by the seashore".

Slovic et al. [17], describe that affect is an important factor in decision making, and state that, "'affect' means the specific quality of 'goodness' or badness' i) experienced as a feeling state (with or without consciousness) and ii) demarcating a positive or negative quality of a stimulus.". Based on this, Ref. [18] suggests that affect is bipolar, and that we therefore should be able to express our preferences on such a scale.

The scale we use is the bipolar univariate model [18]. A bipolar univariate scale can typically range from $[-1,1]$, and is divided into three sub-intervals each associated with one type of affect; negative, neutral, and positive. Positions on the scale with a value lower than zero are associated with negative affect and the zero value is interpreted as neutral affect. Values greater than zero are associated with positive affect.

In our design, each stakeholder's preferences with respect to each action are expressed on this scale. The midpoint (neutral affect) is called the neutrality threshold $t$, communicating that the actions placed there are not considered to improving the focus area, nor to be counterproductive. This enables us to partition the stakeholders into two groups, one group holding the stakeholders with negative affect and one with the stakeholders with positive affect towards the action with respect to each focus area.

\section{B. Measuring Stakeholder Disagreement}

We assume a finite set of actions $\boldsymbol{A}=\left\{A_{1}, A_{2}, \ldots, A_{m}\right\}$, and that an action $A_{i} \in \boldsymbol{A}$ is evaluated against a set of mutually preferentially independent criteria $\boldsymbol{G}=\left\{G_{1}, G_{2}, \ldots, G_{l}\right\} \quad$. $\quad$ Each stakeholder $S_{j} \in S=\left\{S_{1}, \ldots, S_{n}\right\}$ is to assess the value $v_{i k}^{j}$ of each action $A_{i}$ under each criterion $\mathrm{G}_{\mathrm{k}}$ and the weight $w_{k}^{j}$ of $G_{k}$, such that $w_{k}^{j} \geq 0$ and $\sum_{k=1}^{l} w_{k}^{j}=1$. The additive value function in Eq. (1.1) is then used to obtain the utilitarian value of an action $A_{i}$ by summing up the weighted values from all stakeholders.

$$
V\left(A_{i}\right)=\sum_{j=1}^{n} \sum_{k=1}^{l} w_{k}^{j} v_{i k}^{j}
$$

Note that we do not normalize the value by the number of stakeholders. We instead add each stakeholder's value for an action into an overall estimation, or in the words of Bentham [19] "The interest of the community then is, what?-the sum of the interests of the several members who compose it.".

Further, for each action $A_{i}$ we form two stakeholder subsets called the con-group $R_{i k}^{-}$and the pro-group $R_{i k}^{+}$, such that the stakeholders of $R_{i k}^{-}$assign a value of $A_{i}$ with respect to $G_{k}$ lower than the neutrality threshold and that the stakeholders of $R_{i k}^{+}$assign a value at the neutrality threshold or greater than it, see Eq. (1.2).

$$
\begin{aligned}
& R_{i k}^{-}=\left\{S_{j} \in S: v_{i k}^{j}<t\right\}_{1 \leq j \leq n} \\
& R_{i k}^{+}=\left\{S_{j} \in S: v_{i k}^{j} \geq t\right\}_{1 \leq j \leq n}
\end{aligned}
$$

Having this information, for each action $A_{i}$, we create two value ranges for each criterion $G_{k}$. one range for each of the two groups. These are labeled as the con-support $S_{C}$ and prosupport $S_{P}$ respectively and are given from the minimum and maximum stakeholder part-worth utility, i.e. the weightvalue products, see (1.3).

$$
\begin{gathered}
S_{C}=\left[\min _{j \mid s_{j} \in R_{k k}^{-}}\left\{w_{k}^{j} v_{i k}^{j}\right\}, \max _{j \mid s_{j} \in R_{i k}^{-}}\left\{w_{k}^{j} v_{i k}^{j}\right\}\right] \\
S_{P}=\left[\min _{j \mid s_{j} \in R_{i k}^{+}}\left\{w_{k}^{j} v_{i k}^{j}\right\}, \max _{j \mid s_{j} \in R_{i k}^{+}}\left\{w_{k}^{j} v_{i k}^{j}\right\}\right]
\end{gathered}
$$

For each action $A_{i}$ and criterion $G_{k}$ we let the average conindex $c_{i k}$ and average pro-index $p_{i k}$ be two quantitative measures of the sets of part-worth utilities in the two stakeholder groups $R_{i k}^{-}$and $R_{i k}^{+}$, these are defined as

$$
\begin{aligned}
& c_{i k}=\sum_{j \mid S_{j} \in R_{u k}^{-}} w_{k}^{j} v_{i k}^{j} /\left|R_{i k}^{-}\right| \\
& p_{i k}=\sum_{j \mid S_{j} \in R_{k k}^{+}} w_{k}^{j} v_{i k}^{j} /\left|R_{i k}^{+}\right|
\end{aligned}
$$

Thus, the average con- and pro-indexes are the arithmetic mean of the part-worth utility for each of the two stakeholder groups. Another interpretation is that the conand pro-indexes represent a conditional expected value of the part-worth utility $x$ such that $c_{i k}=\mathrm{E}(x \mid x<0)$ and $p_{i k}=$ $\mathrm{E}(x \mid x>0)$.

Then $d_{i k}=\left|c_{i k}-p_{i k}\right|$, i.e. the distance between the proindex and the con-index indicate an additive level of disagreement with respect to the performance of the action $A_{i}$ relative to criterion $G_{k}$. This level of disagreement is called the additive disagreement index $d_{i k}$ for action $A_{i}$ under criterion $G_{k}$. The total disagreement index for action $A_{i}$ is denoted with $T_{i}$ and is the sum of all criterion specific disagreement indexes, such that

$$
T_{i}=\sum_{k \mid G_{k} \in G} d_{i k}
$$

To illustrate, assume a set of eight stakeholders who have provided their weight and value statements for action $A_{1}$ of criterion $G_{1}$ and the weight for $G_{1}$ such that

$$
\begin{aligned}
& w_{1}^{1} v_{11}^{1}=-4, w_{1}^{2} v_{11}^{2}=-3, w_{1}^{3} v_{11}^{3}=-3 \\
& w_{1}^{4} v_{11}^{4}=-2, w_{1}^{5} v_{11}^{5}=1, w_{1}^{6} v_{11}^{6}=2 \\
& w_{1}^{7} v_{11}^{7}=3, w_{1}^{8} v_{11}^{8}=3
\end{aligned}
$$


Then the con-group $R_{11}^{-}$and pro-group $R_{11}^{+}$for action $A_{1}$ and criterion $G_{1}$ are $R_{i k}^{-}=\left\{S_{1}, S_{2}, S_{3}, S_{4}\right\} \quad$ and $R_{11}^{+}=\left\{S_{5}, S_{6}, S_{7}, S_{8}\right\}$ and $c_{i k}=-3$ and $p_{i k}=2.25$. See Figure 1

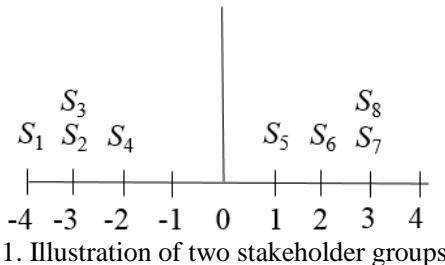

\section{Disagreement Constrained Portfolio Generation}

The portfolio with the highest value while maintaining a predefined resource constraint is given by solving the Knapsack problem [20], in Eq. (1.6). A portfolio $P$ of actions is a subset of all actions $A(P \subseteq A)$. The total portfolio disagreement index is given by summing all actions' total disagreement indexes, see Eq. (1.7). A feasible portfolio has a total portfolio disagreement index lower than the resource constraint $B$, i.e. $T_{p} \leq B$. An action $A_{i}(i\{\in 1, \ldots, m\})$ has an associated value $v_{i}=\mathrm{V}\left(A_{i}\right)$, and a total disagreement index $\mathrm{T}_{\mathrm{i}}$, and an action can either be included $\left(x_{i}=1\right)$ or excluded from the portfolio $\left(x_{i}=0\right)$.

$$
\begin{gathered}
\operatorname{maximize} \sum_{i=1}^{m} v_{i} x_{i} \\
\text { subject to } \sum_{i=1}^{m} T_{i} x_{i} \leq B \\
x_{i} \in\{0,1\}, i=1, \ldots, m \\
T_{p}=\sum_{i \mid A_{i} \in G} T_{i}
\end{gathered}
$$

An efficient portfolio $P$ dominates a portfolio $P^{\prime}$ when it both has a lower total portfolio disagreement index $T_{p}<T_{p}$, and a higher overall utility, $\sum_{\mid A_{i} \in P} \mathrm{~V}\left(A_{i}\right)>\sum_{j \mid A_{j} \in P} \mathrm{~V}\left(A_{j}\right)$.

Efficient portfolios within the range $\left[0, \sum_{i \mid A_{i} \in A} T_{i}\right]$ are generated by the same approach as in Refs. [7], [8]. Eq. (1.6) is calculated for multiple values of $B$, where $B$ in the first run is set to $B=\sum_{i \mid A_{i} \in A} T_{i}$ and for each succeeding run $B$ is set to the total disagreement index value of the latest generated portfolio with a small numerical value subtracted.

\section{A Priori Sensitivity Analysis}

The robustness of the solution can be evaluated by conducting an a priori sensitivity analysis by generating portfolios where the actions disagreement indexes are set to either their minimum or to their maximum values instead of using the average pro- or con-index, see (1.3). This analysis elucidates how the additive disagreement indexes affect the portfolio composition, when they are set to their extreme values. We first denote the border points of the con-support as the minimum con-index $c_{i k}^{-}$and maximum con-index $c_{i k}^{+}$ and for the pro-support, the minimum pro-index $p_{i k}^{-}$and the maximum pro-index $p_{i k}^{+}$. We then define two new additive disagreement indexes for action $A_{i}$ under criterion $G_{k}$, the minimum additive disagreement index $d_{i k}^{-}$(Eq. (1.8)), and the maximum additive disagreement index $d_{i k}^{+}$, (Eq. (1.9)).

$$
\begin{aligned}
& d_{i k}^{-}=\left|c_{i k}^{+}-p_{i k}^{-}\right| \\
& d_{i k}^{+}=\left|c_{i k}^{+}-p_{i k}^{+}\right|
\end{aligned}
$$

The total minimum and maximum disagreement indexes for action $A_{i}$ are then denoted as $T_{i}^{-}$and $T_{i}^{+}$respectively,

$$
\begin{aligned}
& T_{i}^{-}=\sum_{k \mid G_{k} \in G} d_{i k}^{-} \\
& T_{i}^{+}=\sum_{k \mid G_{k} \in G} d_{i k}^{+}
\end{aligned}
$$

The minimum and maximum disagreement portfolios are then generated by Eq. (1.6) with each action's total disagreement index either set to its minimum or maximum.

\section{AN ILLUSTRATIVE EXAMPLE}

Web-based questionnaires are one approach to reaching a large number of citizens to elicit opinions regarding actions under decision. Such a questionnaire is ideally structured so that each question corresponds to one criterion. The performance of an action with regard to a criterion can then be evaluated under each question. Since affect has a bipolar nature [18], it is natural to let the stakeholders input negative, neutral or positive preferences. This makes it possible to measure disagreement among stakeholders, i.e., to identify actions that might lead to conflicts. Further, in a situation where the goal is to select a subset of all actions, we can use this disagreement measure to create portfolios with varying degree of disagreement. In the following small-scale example (A, B and C), we illustrate the use of this approach. Here, the questionnaire consists of two questions (criteria) under which six actions are evaluated by ten stakeholders.

\section{A. Generation of Portfolios}

The procedure consists of four steps; i) preference elicitation, ii) calculation of each action's associated value and its con- and pro-indexes, iii) calculation of each action's associated disagreement, iv) generation of portfolios.

In the first step, ten stakeholders $\left\{S_{1}, \ldots, S_{10}\right\}$ estimate the value $v$ of the actions with regard to the two criteria, $G_{1}$ and $\mathrm{G}_{2}$. The elicitation is conducted using a bipolar univariate scale ranging from, 0 to 100 , with a neutrality threshold at 50. See Table I for the stakeholders' preferences. 
TABLE I: THE TEN STAKeHOLdER'S EVALUATION OF EACH ACTION WITH REGARD TO CRITERION $G_{l}$ AND $G_{2}$

\begin{tabular}{|c|c|c|c|c|c|c|c|c|c|c|c|c|}
\hline \multirow{2}{*}{ Stakeholder } & \multicolumn{10}{|c|}{ Value } \\
\cline { 2 - 13 } & $\boldsymbol{V}_{\mathbf{1 1}}$ & $\boldsymbol{V}_{\mathbf{1 2}}$ & $\boldsymbol{V}_{\mathbf{2 1}}$ & $\boldsymbol{V}_{\mathbf{2 2}}$ & $\boldsymbol{V}_{\mathbf{3 1}}$ & $\boldsymbol{V}_{\mathbf{3 2}}$ & $\boldsymbol{V}_{\mathbf{4 1}}$ & $\boldsymbol{V}_{\mathbf{4 2}}$ & $\boldsymbol{V}_{\mathbf{5 1}}$ & $\boldsymbol{V}_{\mathbf{5 2}}$ & $\boldsymbol{V}_{\mathbf{6 1}}$ & $\boldsymbol{V}_{\mathbf{6 2}}$ \\
\hline$S_{1}$ & 75 & 70 & 80 & 40 & 65 & 40 & 85 & 30 & 40 & 15 & 65 & 85 \\
\hline$S_{2}$ & 15 & 35 & 15 & 15 & 60 & 85 & 85 & 25 & 65 & 80 & 70 & 20 \\
\hline$S_{3}$ & 25 & 75 & 70 & 20 & 90 & 75 & 90 & 25 & 20 & 30 & 30 & 30 \\
\hline$S_{4}$ & 45 & 20 & 90 & 70 & 65 & 90 & 20 & 55 & 70 & 55 & 70 & 70 \\
\hline$S_{5}$ & 25 & 10 & 25 & 70 & 45 & 30 & 70 & 80 & 65 & 10 & 25 & 25 \\
\hline$S_{6}$ & 60 & 30 & 60 & 85 & 80 & 75 & 20 & 65 & 80 & 75 & 55 & 20 \\
\hline$S_{7}$ & 80 & 95 & 25 & 10 & 35 & 95 & 20 & 80 & 15 & 80 & 35 & 70 \\
\hline$S_{8}$ & 25 & 15 & 75 & 85 & 45 & 40 & 70 & 30 & 25 & 30 & 5 & 25 \\
\hline$S_{9}$ & 15 & 80 & 35 & 75 & 15 & 75 & 85 & 25 & 40 & 20 & 60 & 60 \\
\hline$S_{10}$ & 30 & 65 & 75 & 95 & 20 & 75 & 20 & 95 & 85 & 80 & 65 & 10 \\
\hline
\end{tabular}

In the second step we calculate the value of each action and the con- and pro-indexes for each action. Each action's value is given by Eq. (1.1). The calculation of the con- and pro-indexes is divided into two steps. First, for each alternative and criterion we divide the stakeholder set $S$ into two disagreeing stakeholder subsets $R_{i k}^{-}$and $R_{i k}^{+}$(Eq. (1.2)). For example, the con-group and pro-group for action $A_{1}$ under criterion $G_{l}$ are

$$
\begin{aligned}
& R_{11}^{-}=\left\{S_{2}, S_{3}, S_{4}, S_{5}, S_{8}, S_{9}, S_{10}\right\} \\
& R_{11}^{+}=\left\{S_{1}, S_{6}, S_{7}\right\}
\end{aligned}
$$

Second, we calculate the con-index $c_{11}$ and the pro-index $p_{11}$ of each of the two groups. For ease of presentation, we assume that both criteria are equally weighted such that $w_{1}^{j}=w_{2}^{j}=0.5$. The con-index and the pro-index are then given by Eq. (1.4), leading to $c_{11}=12.86$ and $p_{11}=35.83$ in this example. The results are presented in Table II.

\begin{tabular}{|c|c|c|c|c|c|}
\hline \multicolumn{6}{|c|}{ REGARD TO CRITERION $G_{1}$ AND $G_{2}$} \\
\hline & $c_{i k}$ & $p_{i k}$ & $d_{i k}$ & $T_{i}$ & $V\left(A_{i}\right)$ \\
\hline$V_{11}$ & 12.86 & 35.83 & 22.98 & \multirow{2}{*}{50.48} & \multirow{2}{*}{445} \\
\hline$V_{12}$ & 11.00 & 38.50 & 27.5 & & \\
\hline$V_{21}$ & 12.50 & 37.50 & 25.0 & \multirow{2}{*}{54.38} & \multirow{2}{*}{279} \\
\hline$V_{22}$ & 10.63 & 40.00 & 29.38 & & \\
\hline$V_{31}$ & 16.00 & 36.00 & 20.00 & \multirow{2}{*}{42.38} & \multirow{2}{*}{300} \\
\hline$V_{32}$ & 18.33 & 40.71 & 22.38 & & \\
\hline$V_{41}$ & 10.00 & 40.42 & 30.42 & \multirow{2}{*}{54.42} & \multirow{2}{*}{269} \\
\hline$V_{42}$ & 13.50 & 37.50 & 24.00 & & \\
\hline$V_{51}$ & 14.00 & 36.50 & 22.50 & \multirow{2}{*}{49.00} & \multirow{2}{*}{245} \\
\hline$V_{52}$ & 10.50 & 37.00 & 26.50 & & \\
\hline$V_{61}$ & 11.88 & 32.08 & 20.21 & \multirow{2}{*}{45.00} & \multirow{2}{*}{224} \\
\hline$V_{62}$ & 10.83 & 35.63 & 24.79 & & \\
\hline
\end{tabular}

TABLE II: THE TEN STAKEHOLDER'S EVALUATION OF EACH ACTION WiTH

In the third step we calculate the total disagreement for each action (Eq. (1.5)). The additive disagreement $d_{i k}$ for action $A_{i}$ and criterion $G_{k}$ is given by the distance between the con- and pro-indices $\left|c_{i k}-p_{i k}\right|$. For instance, the additive disagreement for action $A_{1}$ and $G_{1}$, is $d_{11}=\left|c_{11}-p_{11}\right|=|12.86-35.83|=22.98$. Then all additive disagreement indices for action $A_{i}$ are summed into the total disagreement index $T_{i}$ (Eq. (1.5)). For action $A_{1}, T_{1}$ becomes 50.48 . The results for the criteria and actions are presented in Table II.

In the fourth step we generate a set of portfolios by solving the Knapsack problem in Eq. (1.6) for multiple values of $B$. In the first run, $B$ is set to $B=\sum_{i \mid A_{i} \in A} T_{i}=295.66$, in the following runs $B$ is re-set to a value of 0.01 disagreement units less than previous portfolio's total disagreement $T_{p}$, i.e., $B=T_{p}-0.01$. Table III shows the 18 portfolios, including both the empty and the full portfolio, and the respective portfolios composition of actions. The value 0 denotes that the current action is not included in the portfolio and 1 denotes that the action is included. Note that the portfolio composition changes when the additive disagreement increases. The overall portfolio utility and disagreement decrease towards the empty portfolio.

\section{B. Analysis of Results}

We can now evaluate the robustness of the generated portfolios, for instance by using the concept of the Core Index (CI) [4], [5]. CI describes an action's degree of inclusion in a set of portfolios, in the range zero to one. A CI of zero denotes an exterior action that is not included in any portfolio. An action included in all portfolios is denoted as a core action and has a CI of one, and an action with a CI in

\begin{tabular}{|c|c|c|c|c|c|c|c|c|}
\hline \multirow{2}{*}{ Portfolio } & \multicolumn{6}{|c|}{ Actions } & \multirow{2}{*}{$\mathbf{V}\left(A_{i}\right)$} & \multirow{2}{*}{$T_{p}$} \\
\hline & $A_{1}$ & $A_{2}$ & $A_{3}$ & $A_{4}$ & $A_{5}$ & $A_{6}$ & & \\
\hline$P_{1}$ & 1 & 1 & 1 & 1 & 1 & 1 & 1762 & 295.66 \\
\hline$P_{2}$ & 1 & 1 & 1 & 1 & 1 & 0 & 1538 & 250.66 \\
\hline$P_{3}$ & 1 & 1 & 1 & 1 & 0 & 1 & 1517 & 246.66 \\
\hline$P_{4}$ & 1 & 1 & 1 & 0 & 1 & 1 & 1493 & 241.24 \\
\hline$P_{5}$ & 1 & 1 & 1 & 1 & 0 & 0 & 1293 & 201.66 \\
\hline$P_{6}$ & 1 & 1 & 1 & 0 & 1 & 0 & 1269 & 196.24 \\
\hline$P_{7}$ & 1 & 1 & 1 & 0 & 0 & 1 & 1248 & 192.24 \\
\hline$P_{8}$ & 1 & 0 & 1 & 0 & 1 & 1 & 1214 & 186.86 \\
\hline$P_{9}$ & 1 & 1 & 1 & 0 & 0 & 0 & 1024 & 147.24 \\
\hline$P_{10}$ & 1 & 0 & 1 & 0 & 1 & 0 & 990 & 141.86 \\
\hline$P_{11}$ & 1 & 0 & 1 & 0 & 0 & 1 & 969 & 137.86 \\
\hline$P_{12}$ & 0 & 0 & 1 & 0 & 1 & 1 & 769 & 136.38 \\
\hline$P_{13}$ & 1 & 0 & 1 & 0 & 0 & 0 & 745 & 92.86 \\
\hline$P_{14}$ & 0 & 0 & 1 & 0 & 1 & 0 & 545 & 91.38 \\
\hline$P_{15}$ & 0 & 0 & 1 & 0 & 0 & 1 & 524 & 87.38 \\
\hline$P_{16}$ & 1 & 0 & 0 & 0 & 0 & 0 & 445 & 50.48 \\
\hline$P_{17}$ & 0 & 0 & 1 & 0 & 0 & 0 & 300 & 42.38 \\
\hline$P_{18}$ & 0 & 0 & 0 & 0 & 0 & 0 & 0 & 0 \\
\hline Core Index & 0.75 & 0.44 & 0.94 & 0.19 & 0.44 & 0.44 & & \\
\hline
\end{tabular}
between zero and one is denoted as a borderline action. 
In the example, all generated actions are borderline actions, see Table III. The core index calculations did not include the portfolio with all actions and the empty portfolio. The two actions with the highest core index are $A_{3}$ with CI 0.94 , and $A_{1}$ and with CI 0.75 , followed by action $A_{2}, A_{5}$ and $A_{6}$, and with CI 0.44 , and $A_{4}$ with CI 0.19 .

\section{Sensitivity Analysis}

To further analyze the robustness of the results, we can conduct an a priori sensitivity analysis. First, for each action $A_{i}$ under each criterion $G_{k}$ we determine the minimum conindex $c_{i k}^{-}$, maximum con-index $c_{i k}^{+}$, minimum pro-index $p_{i k}^{-}$ and the maximum pro-index $p_{i k}^{+}$(Eq. (1.3)). Second, we calculate the minimum additive disagreement index $d_{i k}^{-}$(Eq. (1.8)), and the maximum additive disagreement index $d_{i k}^{+}$ (Eq. (1.9)). We then apply Eq. (1.10) to sum up the criterion specific disagreement indexes into the action's total minimum disagreement index $T_{i}^{-}$and maximum disagreement index $T_{i}^{+}$. The resulting indexes can be seen in Table IV showing the minimum and maximum con-index, minimum and maximum pro-index, minimum and maximum additive disagreement index, and the minimum and maximum total disagreement index.

\begin{tabular}{|c|c|c|c|c|c|c|c|c|}
\hline & $c_{i k}^{-}$ & $c_{i k}^{+}$ & $p_{i k}^{-}$ & $p_{i k}^{+}$ & $d_{i k}^{-}$ & $d_{i k}^{+}$ & $T_{i}^{-}$ & $T_{i}^{+}$ \\
\hline$V_{11}$ & 7.50 & 22.50 & 30.00 & 40.00 & 7.50 & 32.50 & \multirow{2}{*}{22.50} & \multirow{2}{*}{75.00} \\
\hline$V_{12}$ & 5.00 & 17.50 & 32.50 & 47.50 & 15.00 & 42.50 & & \\
\hline$V_{21}$ & 7.50 & 17.50 & 30.00 & 45.00 & 12.50 & 37.50 & \multirow{2}{*}{27.50} & \multirow{2}{*}{80.00} \\
\hline$V_{22}$ & 5.00 & 20.00 & 35.00 & 47.50 & 15.00 & 42.50 & & \\
\hline$V_{31}$ & 7.50 & 22.50 & 30.00 & 45.00 & 7.50 & 37.50 & \multirow{2}{*}{25.00} & \multirow{2}{*}{70.00} \\
\hline$V_{32}$ & 15.00 & 20.00 & 37.50 & 47.50 & 17.50 & 32.50 & & \\
\hline$V_{41}$ & 10.00 & 10.00 & 35.00 & 45.00 & 25.00 & 35.00 & \multirow{2}{*}{37.50} & \multirow{2}{*}{70.00} \\
\hline$V_{42}$ & 12.50 & 15.00 & 27.50 & 47.50 & 12.50 & 35.00 & & \\
\hline$V_{51}$ & 7.50 & 20.00 & 32.50 & 42.50 & 12.50 & 35.00 & \multirow{2}{*}{25.00} & \multirow{2}{*}{70.00} \\
\hline$V_{52}$ & 5.00 & 15.00 & 27.50 & 40.00 & 12.50 & 35.00 & & \\
\hline$V_{61}$ & 2.50 & 17.50 & 27.50 & 35.00 & 10.00 & 32.50 & \multirow[t]{2}{*}{25.00} & \multirow[t]{2}{*}{70.00} \\
\hline$V_{62}$ & 5.00 & 15.00 & 30.00 & 42.50 & 15.00 & 37.50 & & \\
\hline
\end{tabular}

We then generate two sets of portfolios by solving Eq. (1.6) for different values of $B$. The first set is generated by using the actions' minimum disagreement indexes and the second set - by using the actions' maximum disagreement indexes. Table $\mathrm{V}$ shows the set of portfolios generated with each action's total minimum disagreement index. A total of 11 portfolios was generated, including the portfolio where all actions are included and the empty portfolio without any actions. The overall portfolio utility and disagreement decrease towards the empty portfolio. A zero in the table denotes that the action is excluded and 1 that the action is included. The Core Index calculations did not include the portfolio with all actions and the empty portfolio. The actions with the highest core indexes are as previously action $A_{1}$ with CI of one (included in all portfolios), and $A_{3}$ with a CI of 0.89 . This is followed by $A_{2}$ and $A_{5}$ with CI 0.56 , and $A_{6}$ and $A_{4}$ with CI 0.22 .

The second set of portfolios is generated by using the actions' maximum disagreement indexes (Table VI), generating a total of 14 portfolios, including the portfolio where all actions are included and the empty portfolio without any actions. The overall portfolio utility and disagreement decrease towards the empty portfolio. The Core Index calculations did not include the portfolio with all actions and the empty portfolio. Table VI shows that the action with the highest core index is $A_{3}$ with CI 0.92 . This is followed by $A_{1}$ and $A_{4}$ with CI $0.69, A_{5}$ with CI $0.46, A_{2}$ with CI 0.31 , and $A_{6}$ with CI 0.23 .

TABLE V: The Portfolios Generated Using the ACtions’ Minimum

\begin{tabular}{|c|c|c|c|c|c|c|c|c|}
\hline \multirow{2}{*}{ Portfolio } & \multicolumn{6}{|c|}{ Actions } & \multirow{2}{*}{$\mathbf{V}\left(\boldsymbol{A}_{i}\right)$} & \multirow{2}{*}{$T_{p}$} \\
\hline & $A_{1}$ & $A_{2}$ & $A_{3}$ & $A_{4}$ & $A_{5}$ & $A_{6}$ & & \\
\hline$P_{1}$ & 1 & 1 & 1 & 1 & 1 & 1 & 1762 & 162.5 \\
\hline$P_{2}$ & 1 & 1 & 1 & 1 & 1 & 0 & 1538 & 137.5 \\
\hline$P_{3}$ & 1 & 1 & 1 & 0 & 1 & 1 & 1493 & 125 \\
\hline$P_{4}$ & 1 & 1 & 1 & 1 & 0 & 0 & 1293 & 112.5 \\
\hline$P_{5}$ & 1 & 1 & 1 & 0 & 1 & 0 & 1269 & 100 \\
\hline$P_{6}$ & 1 & 0 & 1 & 0 & 1 & 1 & 1214 & 97.5 \\
\hline$P_{7}$ & 1 & 1 & 1 & 0 & 0 & 0 & 1024 & 75 \\
\hline$P_{8}$ & 1 & 0 & 1 & 0 & 1 & 0 & 990 & 72.5 \\
\hline$P_{9}$ & 1 & 0 & 1 & 0 & 0 & 0 & 745 & 47.5 \\
\hline$P_{10}$ & 1 & 0 & 0 & 0 & 0 & 0 & 445 & 22.5 \\
\hline$P_{11}$ & 0 & 0 & 0 & 0 & 0 & 0 & 0 & 0 \\
\hline Core Index & 1.00 & 0.56 & 0.89 & 0.22 & 0.56 & 0.22 & & \\
\hline
\end{tabular}

TABLE VI: The Portfolios Generated Using THE MAXIMUM TOTAL DISAGREEMENT INDEX

\begin{tabular}{|c|c|c|c|c|c|c|c|c|}
\hline \multirow{2}{*}{ Portfolio } & \multicolumn{6}{|c|}{ Actions } & \multirow{2}{*}{$\mathbf{V}\left(A_{i}\right)$} & \multirow{2}{*}{$T_{p}$} \\
\hline & $A_{1}$ & $A_{2}$ & $A_{3}$ & $A_{4}$ & $A_{5}$ & $A_{6}$ & & \\
\hline$P_{1}$ & 1 & 1 & 1 & 1 & 1 & 1 & 1762 & 435 \\
\hline$P_{2}$ & 1 & 1 & 1 & 1 & 1 & 0 & 1538 & 365 \\
\hline$P_{3}$ & 1 & 0 & 1 & 1 & 1 & 1 & 1483 & 355 \\
\hline$P_{4}$ & 1 & 1 & 1 & 1 & 0 & 0 & 1293 & 295 \\
\hline$P_{5}$ & 1 & 0 & 1 & 1 & 1 & 0 & 1259 & 285 \\
\hline$P_{6}$ & 0 & 0 & 1 & 1 & 1 & 1 & 1038 & 280 \\
\hline$P_{7}$ & 1 & 1 & 1 & 0 & 0 & 0 & 1024 & 225 \\
\hline$P_{8}$ & 1 & 0 & 1 & 1 & 0 & 0 & 1024 & 215 \\
\hline$P_{9}$ & 0 & 0 & 1 & 1 & 1 & 0 & 814 & 210 \\
\hline$P_{10}$ & 1 & 0 & 1 & 0 & 0 & 0 & 745 & 145 \\
\hline$P_{11}$ & 0 & 0 & 1 & 1 & 0 & 0 & 569 & 140 \\
\hline$P_{12}$ & 1 & 0 & 0 & 0 & 0 & 0 & 445 & 75 \\
\hline$P_{13}$ & 0 & 0 & 1 & 0 & 0 & 0 & 300 & 70 \\
\hline$P_{14}$ & 0 & 0 & 0 & 0 & 0 & 0 & 0 & 0 \\
\hline Core Index & 0.69 & 0.31 & 0.92 & 0.69 & 0.46 & 0.23 & & \\
\hline
\end{tabular}


We can extend this analysis by calculating the CI over all three sets of portfolios. The action with the overall highest CI is then $A_{3}$ with CI 0.92 followed by $A_{1}$ with CI $0.78, A_{5}$ with CI $0.46, A_{2}$ with CI $0.41, A_{4}$ with CI 0.35 and $A_{6}$ with CI 0.30 .

\section{CONCLUDING REMARKS}

We suggested an approach to participatory group portfolio decision analysis, where information about preferences from diverse stakeholders is present, and the identification portfolios with different levels of disagreement are of interest. For each action that could be part of the portfolio, the stakeholders are divided into two groups for each criterion. The partition is based upon the stakeholders' opinions as of whether they believe the action contributes to the decision objective or if it is counter productive. The distance between the preferences of the two groups on the criterion level is captured by the additive disagreement index, aggregated into a total disagreement index for each action. The efficient portfolios are generated by solving a sequence of Knapsack problems, where for each run the value of the resource constraint is decreased to a value slightly lower than the sum of the previous portfolio's total disagreement indexes. The stability of the solution is then evaluated by a priori sensitivity analysis.

Of concern for future work is to incorporate disagreement measures in multi-stakeholder decision problems, especially in urban planning decision situations, and the development of an approach for a priori sensitivity analysis. The approach is to be complemented with supportive features enabling for gathering of preference information from stakeholders. The preference information in the form of action values and criteria weights can be gathered through web-based questionnaires, and the interaction among the stakeholders and the decision makers can be conducted with web-based approach. The method is then to be implemented in ICT tools for group portfolio decision analysis targeted for use in the public sector. The approach can then be applied in a case study of public planning, and involve stakeholders in the decision process for the identification of planned courses of direction that will cause controversy among the stakeholders or citizens.

\section{ACKNOWLEDGMENT}

This research is partly funded by The Swedish Research Council Formas, grant 2011-3313-20412-31. The authors would also like to thank Lisa Brouwers for valuable comments which helped to improve the paper.

\section{REFERENCES}

[1] M. Danielson, L. Ekenberg, J. Idefeldt, and A. Larsson, "Using a software tool for public decision analysis: The case of Nacka municipality," Decision Analysis, vol. 4, no. 2, pp. 76-90, Jun. 2007.

[2] M. Danielson, L. Ekenberg, A. Ekengren, T. Hökby, and J. Lidén, "Decision process support for participatory democracy," Journal of Multi-Criteria Decision Analysis, vol. 15, no. 1-2, pp. 15-30, 2008.

[3] K. Hansson, G. Cars, M. Danielson, L. Ekenberg, and A. Larsson, "Diversity and public decision making," in Proc. the 2012 International Conference on e-Democracy and Open Government, 2012, pp. 1678-1683.
[4] J. Liesiö, P. Mild, and A. Salo, "Preference programming for robust portfolio modeling and project selection," European Journal of Operational Research, vol. 181, no. 3, pp. 1488-1505, Sep. 2007.

[5] J. Liesiö, P. Mild, and A. Salo, "Robust portfolio modeling with incomplete cost information and project interdependencies," European Journal of Operational Research, vol. 190, no. 3, pp. 679695, Nov. 2008

[6] A. Salo, J. Keisler, and A. Morton, "An invitation to portfolio decision analysis," in Portfolio Decision Analysis, vol. 162, A. Salo, J. Keisler, and A. Morton, Eds. Springer New York, 2011, pp. 3-27.

[7] J. C. Lourenço, A. Morton, and C. A. Bana e Costa, "PROBE - A multicriteria decision support system for portfolio robustness evaluation," Decision Support Systems, vol. 54, no. 1, pp. 534-550, Dec. 2012.

[8] T. Fasth and A. Larsson, "Portfolio decision analysis in vague domains," in Proc. the 2012 IEEE International Conference on Industrial Engineering and Engineering Management, Hong Kong Convention and Exhibition Centre, 10 to 13 December, pp. 61-65.

[9] T. Fasth and A. Larsson, "Sensitivity analysis in portfolio interval decision analysis," presented at The 26th International \{FLAIRS\} Conference, May 22 - 24, 2013 St. Pete Beach, Florida, USA.

[10] M. Danielson, L. Ekenberg, A. Larsson, and M. Riabacke, "Transparent public decision making: Discussion and case study in Sweden," in e-Democracy, D. R. Insua and S. French, Eds. Springer Netherlands, 2010, pp. 263-281.

[11] A. Salo and R. P. Hämäläinen, "Multicriteria decision analysis in group decision processes," in Handbook of Group Decision and Negotiation, vol. 4, D. M. Kilgour and C. Eden, Eds. Dordrecht: Springer Netherlands, 2010, pp. 269-283.

[12] E. Vilkkumaa, A. Salo, and J. Liesiö, "Multicriteria portfolio modeling for the development of shared action agendas," Group Decision and Negotiation, pp. 1-22, 2013.

[13] M. Danielson and L. Ekenberg, "A framework for analysing decisions under risk," European Journal of Operational Research, vol. 104, no. 3, pp. 474-484, Feb. 1998.

[14] M. Danielson, L. Ekenberg, J. Johansson, and A. Larsson, "The DecideIT decision tool," in Proc. ISIPTA, 2003, vol. 3, pp. 204-217.

[15] D. M. Kilgour, Y. Chen, and K. W. Hipel, "Multiple criteria approaches to group decision and negotiation," in Trends in Multiple Criteria Decision Analysis, M. Ehrgott, J. R. Figueira, and S. Greco, Eds. Springer US, 2010, pp. 317-338.

[16] A. Ishizaka and P. Nemery, "A multi-criteria group decision framework for partner grouping when sharing facilities," Group Decis Negot, vol. 22, no. 4, pp. 773-799, Apr. 2012.

[17] P. Slovic, M. L. Finucane, E. Peters, and D. G. MacGregor, "The affect heuristic," European Journal of Operational Research, vol. 177, no. 3, pp. 1333-1352, Mar. 2007.

[18] M. Grabisch, S. Greco, and M. Pirlot, "Bipolar and bivariate models in multicriteria decision analysis: Descriptive and constructive approaches," International Journal of Intelligent Systems, vol. 23, no. 9, pp. 930-969, 2008.

[19] J. Bentham, An Introduction to the Principles of Morals and Legislation, Oxford: Clarendon Press, 1907.

[20] S. Martello and P. Toth, Knapsack Problems: Algorithms and Computer Implementations. J. Wiley \& Sons, 1990.

Tobias Fasth was born in Stockholm, Sweden, in 1980. He received his M.Sc. in computer and systems sciences from Stockholm University in 2013. He is currently a Ph.D. student at the Department of Computer and Systems Sciences (DSV) at Stockholm University. His research interest is decision analysis with a focus on public decision making.

Aron Larsson was born in Umeå, Sweden, in 1976. He received his M.Sc. in computer engineering and his $\mathrm{PhD}$ in computer and systems sciences from Mid Sweden University in 2004 and 2008 respectively. In 2013 he became associate professor at Stockholm University. His research interests include methods, procedures, and applications for/of computer aided risk and decision analysis, especially with incomplete and/or imprecise information. Much of the work has been devoted to development of the decision analysis software DecideIT. Projects include, e.g., process models and methods for public decision making, landmine clearance activities, procurement processes, automated decision making and distributed artificial intelligence in wireless networks.

Maria Kalinina was born in St. Petersburg, Russia, in 1969. She received her Lic. Ph. in computer and systems sciences from Stockholm University in 2014. Her research interests are multi objective optizimation and decision analysis with incomplete information. 